\title{
Norois
}

Environnement, aménagement, société

$241 \mid 2016$

Innovation sociale et développement des territoires dans les campagnes européennes

\section{Le programme LEADER en Europe centrale : apprentissage et pratiques de la gouvernance locale dans les campagnes post-socialistes}

The LEADER program in Central Europe: learning experiences and practices of local governance in post-socialist rural areas

Guillaume Lacquement et Pascal Chevalier

\section{(2) OpenEdition}

Journals

Édition électronique

URL : https://journals.openedition.org/norois/6008

DOI : $10.4000 /$ norois. 6008

ISBN : 978-2-7535-5504-4

ISSN : $1760-8546$

Éditeur

Presses universitaires de Rennes

Édition imprimée

Date de publication : 30 décembre 2016

Pagination : 67-83

ISBN : 978-2-7535-5483-2

ISSN : 0029-182X

Référence électronique

Guillaume Lacquement et Pascal Chevalier, « Le programme LEADER en Europe centrale :

apprentissage et pratiques de la gouvernance locale dans les campagnes post-socialistes ", Norois [En ligne], 241 | 2016, mis en ligne le 31 décembre 2018, consulté le 13 janvier 2022. URL : http:// journals.openedition.org/norois/6008; DOI : https://doi.org/10.4000/norois.6008 


Presses
Universitaires
de Rennes
www.pur-editions.fr
Revue en ligne : http://norois.revues.org

\title{
Le programme LEADER en Europe centrale : apprentissage et pratiques de la gouvernance locale dans les campagnes post-socialistes
}

\author{
The LEADER Program in Central Europe: Learning Experiences and Practices of Local \\ Governance in Post-socialist Rural Areas
}

\author{
Guillaume Lacquement $^{* a}$ et Pascal Chevalier ${ }^{b}$
}

\footnotetext{
*Auteur correspondant : Tel : 0468506884

a Professeur de Géographie, Université de Perpignan Via Domitia, UMR CNRS 5281 ART-Dev, Acteurs, Ressources,

Territoires dans le Développement, France. (lacqueme@univ-perp.fr)

b Professeur de Géographie, Université Paul-Valéry - Montpellier 3, UMR CNRS 5281 ART-Dev, Acteurs,

Ressources, Territoires dans le Développement, France. (pascal.chevalier@univ-montp3.fr)
}

Résumé : Cette contribution propose une étude du programme européen LEADER (2007-2013) en tant que processus d’innovation sociale. Elle s'intéresse à son application dans deux régions d'Europe centrale marquées par l'héritage socialiste (Thuringe en Allemagne orientale et Baranya en Hongrie) et les effets de la transition à l'économie de marché introduite au début des années 1990. La diffusion de l'innovation se lit dans l'aptitude des acteurs locaux à organiser des partenariats (les groupes d'action locale ou GAL) et à délimiter des périmètres d'intervention (les régions-LEADER). Les partenariats coordonnent l'action locale de développement par la conception de stratégies et la réalisation de projets concrets. L'approche comparative montre que l'apprentissage et les pratiques de la gouvernance locale sont différenciés. À l'échelle des États, ils dépendent tout d'abord du mode de gestion du territoire et des processus de décentralisation en faveur de l'autonomie locale. À l'échelle locale, l'innovation sociale s'exprime dans la structuration et le fonctionnement des partenariats qui portent l'action de développement. L'analyse des réseaux sociaux selon les méthodes de la sociologie structurale montre que les pratiques de la gouvernance locale sont sensibles aux effets de contexte géographique.

Abstract: This paper proposes a study of the European LEADER program (2007-2013) as a process of social innovation. It focuses on its implementation in two regions of Central Europe marked by their socialist heritage (Thuringia in eastern Germany and Baranya in Hungary) and the effects of the transition to a market economy initiated in the early 1990s. The diffusion of innovation can be seen in the ability of local actors to organize partnerships (local action groups, or LAGs) and delimit intervention areas (LEADER regions). These partnerships coordinate local development action by devising strategies and implementing concrete projects. The comparative approach presented here shows that learning experiences and practices of local governance are differentiated: At the state level, they depend first and foremost on the method of land management and the decentralization processes implemented in favor of local self-government. At the local level, social innovation is reflected in the structure and functioning of the partnerships that foster development action. The social network analysis conducted using methods of structural sociology shows that local-governance practices are sensitive to the effects of geographical context.

Mots clés : innovations territoriales - LEADER - politique agricole - gouvernance - développement local - Europe centrale

Keywords: territorial innovations - LEADER - agriculture policy - governance - local development - Central Europe 


\section{INTRODUCTION}

Cette contribution au dossier thématique propose une étude du programme européen LEADER (2007-2013) ${ }^{1}$ en tant que processus d'innovation sociale. Elle s'intéresse à son application dans deux régions d'Europe centrale marquées par l'héritage socialiste et les effets de la transition à l'économie de marché introduite au début des années 1990 : la Thuringe en Allemagne orientale et le Baranya en Hongrie du sud. Ce processus renvoie à la manière dont se combinent l'introduction d'une nouveauté institutionnelle et la capacité organisationnelle des sociétés locales à le mettre en œuvre dans des campagnes post-socialistes. L'étude se démarque de la vision normative de l'innovation dans le développement territorial, qui tend à réduire les facteurs principaux du changement et de la croissance économiques à l'introduction de techniques nouvelles de production ou de service. Elle privilégie donc les dimensions sociales de l'innovation et considère le processus de production de l'innovation comme un processus social (Cloutier, 2003; Hillier et al., 2004 ; Fontan et al., 2004).

Le programme européen LEADER comprend deux dimensions principales. La première est spatiale et s'exprime par le maillage des régions-LEADER qui forment les périmètres d'application des stratégies de développement. Ces périmètres ont été tracés par des groupes d'action locale ou GAL qui renvoient à la seconde dimension du programme. Les GAL relèvent d'une dimension sociale qui prend corps dans un organe de concertation et de décision. Composés des membres de la société locale, les GAL sont chargés de la conception et de la mise en œuvre des stratégies de développement au sein des périmètres d'intervention. S'interroger sur le programme LEADER comme processus d'innovation sociale (Dargan et Shucksmith, 2008²), revient à se concentrer sur les nouveaux modes de

1. LEADER est un acronyme qui signifie Liaisons Entre Actions de Développement de l'Économie Rurale et désigne depuis 1990 le programme d'intervention de l'Union européenne en faveur des zones rurales. Le programme LEADER constitue depuis 2007 le quatrième axe du pilier rural de la nouvelle politique agricole commune.

2. Les deux auteurs postulent que le processus d'innovation procède avant tout des situations d'apprentissage par les acteurs sociaux de normes et de règles nouvelles. L'apprentissage n'implique pas nécessairement la découverte et l'adoption de techniques nouvelles car le capital scientifique existant peut être recombiné ou adapté en fonction de l'évolution des activités socio-économiques. Par contre, le capital social joue un rôle déterminant dans le processus d'innovation. Les relations entre les acteurs au sein d'un réseau de coopération jouent sur les formes d'apprentissage gouvernance des territoires locaux en Europe et sur leur apprentissage, plus particulièrement dans les pays post-socialistes.

La gouvernance locale désigne un principe de gestion territoriale qui procède d'une inversion des politiques publiques. Le modèle du développement local ou du développement «par le bas » se fonde sur une redistribution des prérogatives et des compétences en matière d'aménagement en faveur des échelons locaux du système territorial. La démarche LEADER participe de cette dynamique politique qui consiste à promouvoir le développement socioéconomique des zones rurales par des initiatives locales organisées par des partenariats entre acteurs et encadrées par des procédures de contractualisation avec les échelons supérieurs du système territorial. Le programme LEADER incarne donc le paradigme du développement endogène. Il suppose l'implication des acteurs locaux dans des réseaux de coopération afin de concevoir et de mettre en œuvre des projets de développement. Ces formes de coopération introduisent des pratiques nouvelles de gestion territoriale qui se réfèrent au paradigme de la « gouvernance locale » dont les principes soustraient l'action publique au monopole des institutions et de l'administration pour la confier à des groupes d'acteurs d'origines et de compétences diverses (Duran, 2001 ; Leloup, Moyart et Pecqueur, 2005 ; Dubois, 2009; Le Gales, 2010).

L'application du programme LEADER dans les pays post-communistes suppose alors l'institution de systèmes d'action locaux dans des États qui ont dû engager depuis la rupture systémique de 1990 des réformes territoriales allant dans le sens de la décentralisation et de l'autonomisation accrue des collectivités locales. L'application du programme LEADER joue donc un rôle important dans la transformation de la gouvernance des territoires. En tant que systèmes d'action locaux, les GAL sont le lieu de l'apprentissage collectif de normes et de règles nouvelles de l'action publique en faveur du développement local (Teisserenc, 1994). Il s'agit d'apprendre des compétences nouvelles, de se familiariser avec de nouvelles procédures et d'organiser en réseaux les nouveaux partenariats (Maurel, Chevalier et Lacquement, 2014).

qui vont conduire à l'institution d'un système d'innovation, comme le programme LEADER par exemple. 
L'étude propose ici une approche comparative qui participe à la recherche des dimensions spatiales de l'innovation. On se pose la question de savoir quels sont les régimes territoriaux favorables à la genèse de l'action locale. À cette fin, on retient dans le cadre de cette contribution, deux contextes territoriaux, la Hongrie et les nouveaux Länder allemands ${ }^{3}$ afin d'explorer l'hypothèse des effets de contexte sur l'application du programme LEADER en tant que processus social d'innovation. D'un point de vue méthodologique, l'enjeu consiste à emprunter l'un des outils d'analyse de la sociologie structurale, pour caractériser le fonctionnement des réseaux de coopération et la manière dont ils s'articulent avec le territoire local.

On reviendra tout d'abord sur la diffusion de cette forme d'innovation en montrant que l'application du programme LEADER dans les pays européens résulte d'un transfert de politique publique dans le cadre d'un processus d'européanisation. On s'intéressera ensuite à sa mise en œuvre par le tracé des périmètres d'intervention et l'institution des groupes d'action locale afin de mettre au jour des logiques différentes d'application du programme. Enfin, l'approche comparative montrera que les pratiques de l'innovation sont différenciées et que l'apprentissage de la démarche LEADER s'inscrit dans un processus territorialisé.

\section{DifFUSION DE L'INNOVATION : LE TRANSFERT DE POLITIQUE PUBLIQUE LEADER}

L'élargissement de l'Union européenne a créé les conditions favorables à la circulation des nouveaux modèles et des nouveaux instruments de l'action publique (Delpeuch, 2008; Maurel, Chevalier et Lacquement, 2014). Se référant au paradigme du « développement local », expérimentés avec succès et mis en œuvre dès le début des années 1990 dans les espaces ruraux de l'Europe occidentale, ceux-ci sont transférés, dans le cadre d'un processus d'européanisation de l'action publique, aux nouveaux États membres ayant jadis appartenu au système

3. Les nouveaux Länder allemands forment la partie orientale de l'Allemagne fédérale. Ils procèdent de l'absorption de l'ex-RDA par la République fédérale dans le cadre du processus de réunification de 1990. L'étude se concentre sur les cinq Länder orientaux, mais les considèrent dans leur contexte contemporain. Les analyses et les représentations cartographiques feront donc référence au pays dans son ensemble. communiste. Aux antipodes du modèle centralisé que connaissaient ces pays avant le changement de système politique, cette démarche a instauré de nouveaux dispositifs d'action publique s'organisant selon une conception du territoire accordant plus de place à la répartition des responsabilités entre les acteurs institutionnels et la société civile, à la mise en œuvre de nouveaux modes de gestion territoriale et à la négociation entre les acteurs situés aux diverses échelles de gouvernance infra et supranationale.

La démarche LEADER, fondée sur une logique ascendante de l'action publique locale (bottom-up), participe de cette dynamique qui consiste à promouvoir le développement socio-économique des zones rurales «par le bas» (Maurel, 2008), c'està-dire porté par des initiatives locales fondées sur des partenariats entre acteurs et encadrées par des procédures de contractualisation avec les différents échelons du système territorial. Construite sur les deux paradigmes du développement endogène et de la gouvernance locale, elle consiste à orienter les efforts et les investissements sur des projets conçus à l'échelle de communautés rurales dans le but de renforcer la capacité d'initiative des acteurs locaux. Elle participe de l'émergence d'un nouveau mode de gouvernance territoriale, inédit dans ces pays, qui tend à vouloir renforcer «la démocratie locale et participative » (Dolowitz et Marsh, 2000). La mécanique de transfert déclenchée par les étapes successives de l'élargissement de l'Union européenne passe par des prismes nationaux et s'inscrit dans des trajectoires institutionnelles.

\section{La contractualisation multi-niveaux}

L'intervention de type LEADER se rapporte au mode d'action publique envisagé dans sa globalité. En tant que politique publique inspirée par l'UE, sa mise en œuvre se situe à l'articulation de divers échelons territoriaux (européen, national, régional et local). Elle concerne ainsi une vaste arène de négociation entre de multiples acteurs politiques et sociaux. En raison des interactions entre divers niveaux de gouvernement et échelons hiérarchiques de l'administration publique, les configurations d'acteurs qui participent au transfert sont complexes (Chevalier et Maurel, 2013). Ils s'organisent en trois niveaux. 
Lacteur «source » supranational, autrement dit l'UE, définit les orientations en matière de politique rurale européenne en inscrivant ses objectifs stratégiques dans le programme de la Politique européenne de développement rural. Cette politique propose un menu de mesures au sein duquel les États membres peuvent choisir des actions pour lesquelles ils bénéficieront d'un soutien financier communautaire dans le cadre de programmes de développement rural intégré. Au niveau national, l'acteur étatique constitue le principal « opérateur» du transfert. Il détermine les objectifs stratégiques de sa politique de développement rural dans un Plan de Développement Rural (PDR) et met en place le dispositif institutionnel de sa gestion. Enfin, au niveau local, les acteurs « receveurs-opérateurs » emmenés par l'administration des GAL sont chargés de mettre au point leur propre stratégie de développement local et de l'appliquer dans le cadre réglementaire défini par «l'opérateur » de transfert. La diffusion de la démarche LEADER se fonde sur une contractualisation à ces trois niveaux qui encadre le processus de transfert.

Au niveau national, les documents de référence de ce transfert sont donc les Plans de Développement Rural (PDR) dont on propose ici une analyse textuelle des chapitres concernant l'application du pro- gramme LEADER. Chaque territoire local dispose, en théorie, d'une grande latitude dans l'interprétation de ces plans et dans les modalités et les formes de mise en ouvre locale du programme LEADER. Néanmoins, la force normative des PDR influence les processus décisionnels et la conception même des stratégies aux échelles locales.

Ces plans, dans leur partie relative au programme LEADER, comprennent de manière uniforme dans chaque pays, cinq chapitres : les objectifs prioritaires nationaux, les modalités de gestion du programme, la définition des territoires pertinents, les critères de sélection des GAL, la méthodologie d'élaboration des stratégies de développement local. On propose ici d'en cibler deux, dans le cadre d'une analyse textuelle, qui éclairent, particulièrement les disparités dans les processus de mise en œuvre (voir encadré méthodologique).

\section{Les Plans de Développement rural au prisme des États}

L'analyse des mesures de l'axe LEADER considérées comme prioritaires dans chaque programme national (c'est-à-dire bénéficiant de la part de financement la plus élevée) conduit à identifier quatre

\section{Encadré méthodologique}

D'un point de vue méthodologique, les priorités des objectifs stratégiques des programmes nationanx ont été identifiées à partir de la recherche de récurrence et du poids de quatre occurrences (correspondant à l'agrégation de plusieurs mots ou groupes de mots dont le sens est relativement proche):

- «qualité de vie» (comprenant les termes agrégés de bien-être, mode de vie, infrastructures, équipements, cadre de vie);

- «compétitivité des territoires " (comprenant les termes d'entreprise, valeur ajoutée, concurrence, compétitivité, emploi);

- «patrimoine naturel et culturel » (comprenant les termes agrégés de nature, environnement, histoire locale, traditions, culture, fêtes);

- «activité touristique rurale» (comprenant les termes agrégés de nuitées, hébergement, gîtes, équipements touristiques).

Les facteurs de délimitation des régions LEADER ont étés identifiés à partir des quatre occurrences suivantes:

- «taille» (comprenant les termes agrégés de densité démographique, nombre d'habitants, superficie);

- «intégration des villes » dans les périmètres (comprenant les termes agrégés d’urbain, agglomération, villes, bassin d'emploi, mobilités résidentielles);

- «homogénéité géographique» (comprenant les termes agrégés de culture, traditions, environnement physique, histoire);

- « articulation avec des maillages existants » (comprenant les termes agrégés de concordance des périmètres, périmètres institutionnels, espaces de gestion). 
modèles nationaux dominants de conception du développement rural ${ }^{4}$ (figure 1).

Si dans la plupart des pays européens les mesures prioritaires sont destinées à améliorer l'économie rurale, et en particulier soutenir le développement des activités non agricoles et la compétitivité, tout comme préserver et valoriser le patrimoine dans le cadre de projets touristiques, dans les pays postsocialistes d'Europe centrale, les priorités semblent bien différentes. La typologie, qui tend à les discriminer nettement, montre que la recherche de l'amélioration de la qualité de vie dans les zones rurales est prioritaire dans l'ensemble des Plans nationaux de développement rural.

Cet objectif comprend des mesures de rénovation des villages, de conservation et de valorisation du patrimoine, mais concerne surtout la modernisation des infrastructures et des équipements ruraux. Ce choix stratégique peut s'expliquer en partie par le sous-équipement des communes rurales dont le retard de développement impose plus qu'ailleurs des investissements matériels et par une conception encore très agro-centrée du développement rural dans un contexte de faible diversification des activités rurales. Dans ces pays, le programme LEADER vient suppléer l'effort des collectivités locales et de l'État dans le financement des opérations d'équipement rural. Les nouveaux Länder allemands pourraient rejoindre ce groupe, mais ils sont englobés dans les objectifs de l'Allemagne fédérale qui privilégient la préservation et la restauration des patrimoines.

Concernant la délimitation des périmètres d'intervention ou des régions LEADER, les pays postsocialistes d'Europe centrale ne se distribuent pas de manière homogène dans la seconde typologie (figure 2). Ils relèvent soit du premier groupe pour lequel la réglementation nationale prescrit l'insertion des régions LEADER dans le réseau des coo-

4. Sur la base des critères élaborés grâce à l'analyse textuelle des réglementations et l'application des algorithmes statistiques, il s'est agi de proposer une répartition des pays en un certain nombre de classes intrinsèquement homogènes et suffisamment distinctes les unes des autres. À cette fin, nous avons choisi la méthode dite «Hybrid clustering » ou « classification mixte » (Lebart et al., 1997) dont l'objectif est de maximiser les variances interclasses tout en minimisant les variances intra-classes. Elle suggère de combiner les deux méthodes de classification classiques - classification ascendante hiérarchique $(\mathrm{CAH})$ et classification autour de centres mobiles (CCM) - afin d'atténuer les inconvénients propres à chaque méthode, en l'occurrence pour la $\mathrm{CAH}$, la perte de « ressemblance » au fur et à mesure des agrégations, et pour la CCM, l'obligation de fixer a priori le nombre de classes et une partition dépendante des centres initiaux choisis.

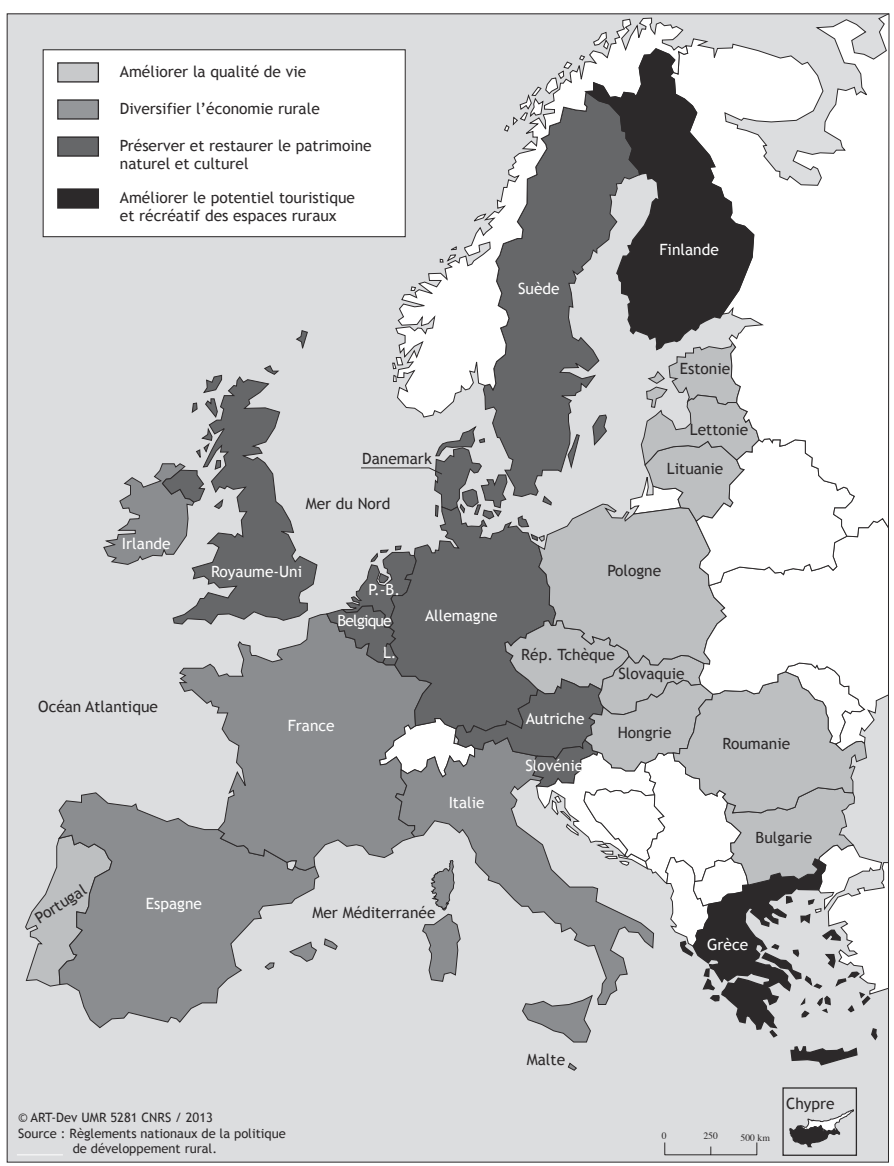

Figure 1 : La définition des objectifs prioritaires du programme LEADER (2007-2013) selon les États membres de l'Union européenne 2013) by EU member state

Definition of the priority objectives of the LEADER program (2007-

pérations intercommunales antérieures ou existantes, soit du quatrième groupe où la réglementation exprime la volonté de consolider le maillage administratif du territoire. C'est notamment le cas en Hongrie où la procédure de création des GAL s'est extrêmement durcie après 2007. Dans tous les cas, on observe un effet de tropisme des maillages institutionnels existants sur lesquels viennent s'insérer les périmètres d'intervention du programme LEADER.

Au final, le mode de transfert de politique publique promu par l'UE accorde aux États membres des marges de manœuvre leur permettant d'adapter l'instrument LEADER aux logiques d'action répondant à leurs objectifs de politique publique. Les prismes nationaux, c'est-à-dire les éléments institutionnels, les acteurs et/ou les facteurs culturels qui sont susceptibles de déterminer la réception des 


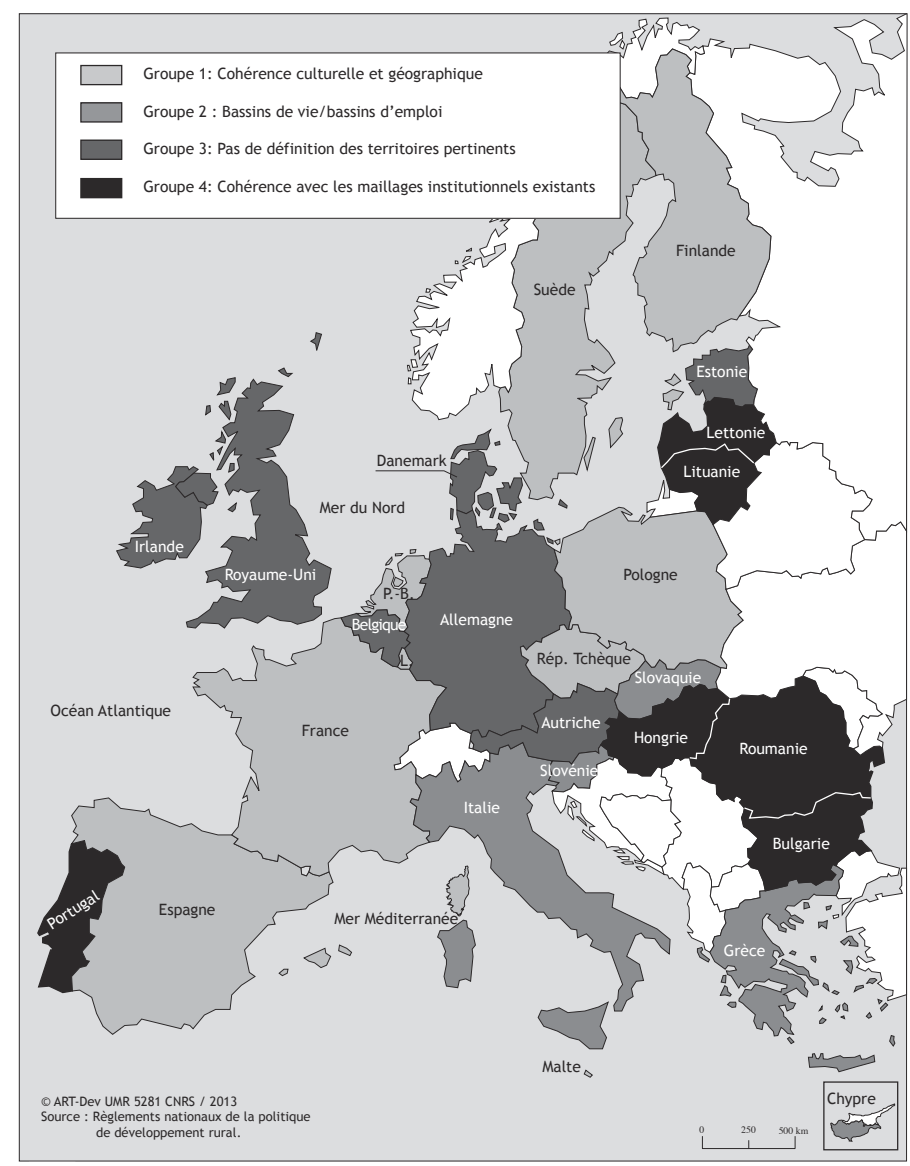

Figure 2 : La délimitation des régions-LEADER (2007-2013) selon les États membres de l'Union européenne

Delimitation of LEADER (2007-2013) regions by EU member state

normes et des politiques européennes constituent un puissant facteur de différenciation. La pression adaptative exercée par l'UE est diversement reçue et intégrée par les prismes nationaux et se traduit par des logiques d'action différenciées. Le processus de transfert peut conduire à une absorption du modèle LEADER proposé par l'UE, ouvrir sur une transformation de celui-ci ou se solder par le rejet du paradigme qui le promeut. La distinction entre une logique d'accompagnement et une logique d'intervention offre une grille d'interprétation de la manière dont l'acteur étatique combine techniques et outils de politique publique pour encadrer la mise en œuvre de l'instrument LEADER. Les pays d'Europe centrale se partagent entre ces deux logiques.

\section{L'APPLICATION DE L'INNOVATION : LOGIQUES D'ACCOMPAGNEMENT ET LOGIQUES D'INTERVENTION}

La logique d'accompagnement est fidèle au paradigme de l'action publique LEADER. Elle procède d'une démarche ascendante qui postule la capacité d'initiative des acteurs locaux, leur aptitude à composer sur la base du volontariat un partenariat et à délimiter un périmètre d'action. À l'échelle nationale, on observe la formation d'un maillage territorial hétérogène ne couvrant que partiellement l'espace rural en raison d'un mode de sélection des projets de GAL fondé sur la compétition (principe de différenciation). C'est le cas dans les nouveaux Länder où l'apprentissage s'est étendu sur près de deux décennies ${ }^{5}$.

La logique d'intervention est l'expression de la volonté de l'acteur étatique de plier l'instrument LEADER aux objectifs spécifiques qui sont les siens. En conséquence, le dispositif institutionnel relève d'un mode de commande et de contrôle dans une démarche top-down. Les procédures définissent des règles dures de formation des partenariats et de détermination des périmètres, sur la base de maillages institutionnalisés. L'intention du pouvoir central est d'imposer la maille convenant le mieux à l'objectif d'instrumentation de la démarche LEADER dans une logique de quasi-planification et de gestion distributive. C'est surtout le cas en Hongrie où la mise en œuvre du programme LEADER est le produit d'un pilotage top-down verrouillant les canaux de diffusion des valeurs du modèle LEADER. En instrumentalisant stratégies et projets au service des politiques municipales, les élites locales expri-

\footnotetext{
5. En croisant les deux logiques d'action qui dépendent du prisme national (logique d'accompagnement et logique d'intervention) et le degré d'appropriation de la démarche LEADER par les populations locales (élargi à une diversité d'acteurs locaux ou limité à une élite), P. Chevalier, M.C. Maurel et P. Pola (2015) définissent trois situations types

- L'absorption du modèle européen. Il est atteint lorsqu'une logique d'accompagnement favorise une large appropriation du modèle LEADER par les acteurs locaux.

- L'adaptation au modèle LEADER. C'est le cas dans des pays moins avancés en matière d'apprentissage qui s'efforcent de surmonter les freins institutionnels dans une logique d'accompagnement ou d'intervention du transfert de modèle (cas tchèque) La participation active des sociétés rurales vient souvent compenser l'influence d'un mode de pilotage top-down.

- L'inertie. Il est le produit d'un pilotage top-down, d'une logique d'intervention verrouillant les canaux de diffusion des valeurs du modèle LEADER. En instrumentalisant stratégies et projets au service des politiques municipales, les élites locales expriment une forme de rejet du paradigme de l'action LEADER (cas hongrois).
} 
ment une forme de rejet du paradigme de l'action LEADER.

L'examen des modalités concrètes de la mise en œuvre du programme LEADER permet de mieux distinguer et de mieux caractériser ces deux logiques. Elles s'appliquent d'une part au tracé des périmètres d'intervention et d'autre part à l'institution des groupes d'action locale ou GAL.

\section{Le tracé des régions LEADER}

En Allemagne fédérale, la mise en œuvre du programme LEADER a été déléguée aux Länder dans le cadre d'une procédure décentralisée. À l'est comme à l'ouest ${ }^{6}$, les États fédérés ont établi des critères variables de superficie et de densité : 30000 à 100000 habitants pour une densité moyenne de 50 habitants $/ \mathrm{km}^{2}$ dans le Land de Brandebourg par exemple ou 50000 à 150000 habitants dans le Land de Thuringe mais sans seuil de densité et avec la possibilité d'inclure des districts urbains (Tietz, 2007). Globalement, la trame des régions LEADER s'est densifiée par rapport aux programmes précédents. Elle traduit les discontinuités de peuplement entre régions urbaines et régions rurales en Allemagne. Elle exprime surtout la volonté politique de promouvoir le développement endogène car la démarche LEADER est clairement conçue comme une mesure d'accompagnement intégrée à la politique de développement rural (Lacquement, 2012). En outre, la dynamique de transfert institutionnel engagée en Allemagne au lendemain de l'unification conduit à une forme de banalisation des territoires ruraux post-socialistes. La procédure d'application du programme LEADER ne distingue pas a priori les régions d'ex-RDA du reste de l'Allemagne. Les campagnes des nouveaux Länder ne bénéficient pas de mesures dérogatoires ou de conditions particulières. Les principes de subsidiarité sont appliqués après un retour rapide au régime administratif commun, qui au début des années 1990, a pris la forme d'une rupture brutale dans le mode de gestion des territoires locaux. En RDA, le pouvoir communal avait été singulièrement réduit par la centralisation du pouvoir politique, les mécanismes de la planification économique mais aussi par le transfert d'une

6. À l'est, les procédures décentralisées ont été introduites après le rétablissement des Länder en 1990 et l'application de la Loi fondamentale de la République fédérale dans le cadre de la réunification. grande partie des prérogatives locales aux structures de production, à savoir les fermes d'État et les coopératives agricoles. La restauration des principes de l'autonomie locale signifiait alors l'assimilation de nouveaux types de savoirs et la mobilisation de nouvelles formes de compétences. Les fonctions courantes de l'administration nécessitaient la réorganisation des services et la formation des personnels. Les missions d'aménagement du territoire local supposaient des choix dans l'affectation des ressources et l'orientation des investissements, ce qui modifiait complètement la manière de concevoir la gestion de l'espace local (Lacquement, 2012).

En Hongrie, la mise en œuvre du programme a été prise en charge par une agence d'État dépendante du ministère de l'Agriculture (l'Agence pour l'Agriculture et le développement rural) et représentée par 19 offices déconcentrés dans les départements (megye). Cette agence a constitué l'unique opérateur de l'application du programme. Ses offices ont créé des Bureaux de développement local dans les micro-régions statistiques du pays, puis ont tracé les périmètres des régions LEADER sur la base du découpage micro-régional. On obtient une couverture quasi exhaustive du pays ainsi qu'un maillage homogène constitué de périmètres de grande taille en coïncidence avec le maillage administratif. Le second pilier de la PAC est ici transcrit de manière à doter le pouvoir central d'outils de contrôle sur l'échelon micro-régional, qui devient un niveau de gestion des services et des équipements publics.

\section{L'institution des groupes d'action locale ou GAL}

Logiques d'accompagnement et d'intervention s'expriment dans le même temps dans la manière d'instituer les groupes d'action locale. Un GAL est d'abord une entité dotée d'un statut juridique et pourvue d'organes de décision et de gestion. Il procède ensuite d'un partenariat fondé sur la mise en réseau d'acteurs sollicités pour leur appartenance à des horizons socioprofessionnels divers (administration, monde socio-économique, sphère associative) (Commission Européenne, 2011). En ce sens, il se présente comme un acteur collectif de l'action publique. La composition des partenariats et leur mode de structuration sont donc un enjeu du déploiement de l'action de développement local 
(Maurel, Chevalier, Lacquement, 2014). Pour comprendre les conditions de la formation des GAL dans les campagnes post-socialistes, on propose d'examiner deux situations locales.

La première intéresse le GAL Henneberger Land, situé en Thuringe occidentale dans l'un des cinq nouveaux Länder allemands. Le mode de composition du partenariat traduit manifestement le souci des équilibres institutionnels et territoriaux. Le GAL réunit une trentaine de membres (tableau 1). Il compte des représentants des deux villes principales, Meiningen et Schmalkalden, qui étaient autrefois les chefs-lieux des deux districts de l'époque socialiste et qui forment aujourd'hui le chef-lieu bicéphale du nouveau district issu de la réforme territoriale de 1992. Il comprend également un élu du district urbain de Suhl car le nouveau dispositif LEADER souhaite intégrer les zones périurbaines et les relations villes-campagnes dans la conception des stratégies de développement local. Il fait enfin une place aux délégués des communes ou des nouveaux groupements intercommunaux. En second lieu, le partenariat s'étend au milieu associatif. Le manager du GAL s'est attaché à solliciter l'ensemble des associations actives et impliquées dans la vie économique et sociale : Croix-Rouge, associations de jeunes, de femmes mais surtout l'association de gestion paysagère, l'association de protection de l'environnement et l'association de développement régional qui, dans le cadre des relations institutionnelles établies avec les administrations locales, ont pris en charge des missions de gestion et d'aménagement de l'espace. Les partenaires relèvent en dernier lieu du monde de l'entreprise. Les chefs d'exploitation agricole sociétaire, c'est-à-dire les dirigeants des structures d'exploitation qui ont pris la succession des coopératives socialistes, occupent une place privilégiée dans le comité de pilotage tandis que

\begin{tabular}{|c|c|c|}
\hline Administrations/Élus & Entreprises & Associations et société civile \\
\hline \multicolumn{3}{|c|}{ Comité directeur (Vorstand) } \\
\hline $\begin{array}{l}\text { - Maire de commune } \\
\text { - Représentant du Kreis }\end{array}$ & $\begin{array}{l}\text { - Chef d'exploitation agricole sociétaire } \\
\text { - Chef d'exploitation agricole sociétaire } \\
\text { (également représentant du syndicat } \\
\text { agricole majoritaire) } \\
\text { - Chef d'entreprise d'une société d'inno- } \\
\text { vation technologique (pépinière d'entre- } \\
\text { prises) (F) }\end{array}$ & - Représentant de l'Église protestante \\
\hline \multicolumn{3}{|c|}{ Comité consultatif (Fachbeirat) } \\
\hline $\begin{array}{l}\text { - Maire de commune } \\
\text { - Maire de commune } \\
\text { - Maire de commune } \\
\text { - Maire de commune } \\
\text { - Maire de commune } \\
\text { - Représentant de la ville de Meinigen } \\
\text { - Représentant de la ville de Schmalkal- } \\
\text { den } \\
\text { - Représentant de la ville de Suhl } \\
\text { - Représentant du Kreis } \\
\text { - Représentante du Kreis (F) } \\
\text { - Représentant de l'office de développe- } \\
\text { ment rural }\end{array}$ & $\begin{array}{l}\text { - Chef d'exploitation agricole sociétaire } \\
\text { - Chef d'exploitation agricole sociétaire } \\
\text { (F) } \\
\text { - Représentant d'une Société de conseil } \\
\text { pour entreprises } \\
\text { - Représentant de la Chambre de com- } \\
\text { merce et d'industrie } \\
\text { - Représentant de la Chambre de l'arti- } \\
\text { sanat } \\
\text { - Représentante de la Caisse d'épargne } \\
\text { dans le Kreis (F) }\end{array}$ & $\begin{array}{l}\text { - Représentant du syndicat agricole } \\
\text { majoritaire } \\
\text { - Représentante d'une association pour } \\
\text { la jeunesse (F) } \\
\text { - Représentant de la Croix rouge de } \\
\text { Schmalkalden } \\
\text { - Responsable d'une association pour le } \\
\text { développement local (F) } \\
\text { - Responsable d'une association de pro- } \\
\text { tection du paysage (F) } \\
\text { - Représentant d'un centre de formation } \\
\text { pour adulte et d'information sur l'envi- } \\
\text { ronnement } \\
\text { - Responsable du Parc naturel régional } \\
\text { (statut associatif) (F) } \\
\text { - Responsable local d'une association de } \\
\text { défense de la nature }\end{array}$ \\
\hline \multicolumn{3}{|c|}{ Manager du Gal (F) } \\
\hline
\end{tabular}

$\mathrm{F}=$ Femme

Source : enquêtes de terrain 2009-2011

Tableau 1 : Composition du Conseil d'administration du GAL Henneberger Land, Land de Thuringe, Allemagne Membership of the board of directors of the Henneberger Land Local Action Group, Land of Thuringia, Germany 
les entreprises des autres branches d'activités sont indirectement représentées par les membres des chambres consulaires, chambres de l'industrie, du commerce et de l'artisanat (Lacquement, 2012).

La seconde situation locale renvoie au cas du GAL Zengö-Duna situé dans le Baranya en Hongrie. La formation des GAL est l'aboutissement d'une procédure longue et complexe qui a été pilotée par l'autorité de management (l'Agence d'État). Des bureaux de développement local établis dans les micro-régions ont eu pour mission de constituer des Communautés locales (helyi közösség, HK), puis de composer leurs comités de pilotage, qui sont devenus après validation administrative, des GAL. Ainsi, le GAL de Zengö-Duna procède de la réunion de deux micro-régions (Mohács et Pécsvárad) (tableau 2). Il compte 215 membres dont les 60 représentants des communes car un règlement national impose la représentation de toutes les collectivités participantes. Le GAL compte également 87 représentants du secteur associatif et 68 représentants du secteur économique, qui pour beaucoup sont des élus locaux, si bien que le principe de représentation des trois secteurs devient un habillage standardisé qui masque la prédominance de fait des acteurs du pouvoir local (qu'on estime dans le cas présent à $56 \%$ ) (Kovách et Kucerová, 2006).

Les deux situations sont très différentes, parce qu'elles sont inscrites dans deux contextes institutionnels qui ont donné lieu à des processus et à des pratiques distincts de décentralisation et de transfert de prérogatives. Au-delà des différences, le mode de composition des GAL peut être interprété comme une sorte de partition de la représentativité qui a conduit à une certaine formalisation de la démarche participative. La représentation formelle des trois secteurs ne détermine pas a priori l'implication des acteurs dans le réseau de coopération et ne préjuge pas de l'efficience de la gouvernance locale. Pour saisir les pratiques nouvelles de gouvernance inspirées par la démarche LEADER, il est nécessaire d'analyser le fonctionnement des partenariats constitués en réseaux.

\section{LES PRATIQUES DE L'INNOVATION : UN APPRENTISSAGE TERRITORIALISÉ}

Le fonctionnement des GAL peut se mesurer aux liens qu'entretiennent les individus au sein du réseau de coopération. On considère en effet que ce sont les liens entre les individus qui structurent le système d'action local. Plus que la position des individus dans la société locale, la qualité et l'intensité des liens entre les individus influencent la capacité d'action du GAL à impulser des projets de développement, jouent sur les mécanismes de concertation et de décision et donc de coordination de l'action publique. C'est donc au sein d'un réseau que le groupe entreprend l'apprentissage d'un registre d'action et d'un mode de gouvernance (Maurel, Chevalier et Lacquement, 2014).

Pour étudier la nature des relations entre les membres des GAL, nous avons emprunté les méthodes de la sociologie structurale (Mercklé, 2011). Le postulat structuraliste considère le fonctionnement des réseaux sociaux, non pas à partir de la somme des relations qui s'établissent entre les individus mais à partir de la nature de ces relations qui peuvent être plus ou moins denses, plus ou moins équivalentes, plus ou moins connexes c'est-àdire interdépendantes (Forsé, 2008). Les liens qui s'entrecroisent dans le réseau forment une matrice de ressources sociales qui contribue au capital social des individus et crée une sorte de valeur ajoutée qui majore leur capacité d'action (Lin, 1995). La nature et l'intensité des liens confèrent alors aux

\begin{tabular}{|l|c|c|c|c|}
\hline & Secteur public & Secteur associatif & Secteur privé & Total \\
\hline Nombre total de membres & $60(28 \%)$ & $87(40 \%)$ & $68(32 \%)$ & $215(100 \%)$ \\
\hline Représentation selon le statut officieux & $121(56,3 \%)$ & $55(25,6 \%)$ & $39(18,1 \%)$ & 215 \\
\hline $\begin{array}{l}\text { Répartition selon les composantes } \\
\text { territoriales }\end{array}$ & $60(100 \%)$ & $87(100 \%)$ & $68(100 \%)$ & 215 \\
\hline Microrégion de Mohács (Bóly) & $40(66 \%)$ & $58(66 \%)$ & $58(85 \%)$ & 156 \\
\hline Microrégion de Pécsvarád & $20(34 \%)$ & $29(34 \%)$ & $10(15 \%)$ & 59 \\
\hline
\end{tabular}

Tableau 2 : Composition du conseil d'Administration du GAL Zengő-Duna, Hongrie (Sources : comité de pilotage du GAL Zengő-Duna enquêtes de terrain 2009-2013)

Membership of the board of directors of the Zengö-Duna Local Action Group, Hungary 
différents individus une position plus ou moins centrale. La méthode d'analyse s'appuie sur les résultats d'enquêtes par questionnaire pour construire des graphes de relations qui permettent d'interpréter la structure des réseaux ${ }^{7}$.

\section{Système d'action local et liens d'interconnaissance}

La structure des réseaux peut se lire à partir des liens d'interconnaissance établis entre les individus. Les partenariats qui sont à l'origine de la création des GAL sont issus de relations d'interconnaissance que le programme européen LEADER institutionnalise dans une entité nouvelle. Ce processus fonde le système d'action local, qui d'un GAL à l'autre, présente des configurations très différentes que l'approche monographique permet de restituer.

Dans le GAL situé dans les nouveaux Länder allemands, la situation d'interconnaissance se caractérise par la densité et la connexité des relations interpersonnelles. Le nombre d'arcs (liens) entre les sommets (individus) est élevé et se développe par l'intermédiaire de chaînes qui créent des liens supplémentaires entre les individus. Le tissu de relations est donc faiblement hiérarchisé. On observe cependant des individus qui polarisent davantage que d'autres le système d'interconnaissance. Ce sont des maires ou des élus locaux, ainsi que des chefs d'entreprise comme les chefs des exploitations agricoles sociétaires, les exploitations agricoles qui ont pris au début des années 1990 la succession juridique des coopératives socialistes. Ces personnes doivent leur notoriété aux activités et aux responsabilités qu'elles exercent au niveau local et qui tendent à multiplier les opportunités de contacts interpersonnels (Lacquement, 2012) (figure 3). Elles peuvent faire partie de l'ancienne génération, celle des cadres formés par le régime socialiste dans les années 1980, et ayant conduit avec succès la reconversion de leurs activités dans le cadre du changement de système. Leur influence est grande sur la génération nouvelle,

7. Pour évaluer la nature de la relation entre les individus, il a d'abord été procédé à une enquête visant à mettre en lumière la structure d'interaction des membres des GAL puis à la réalisation d'une analyse dite des réseaux sociaux (social network analysis). Au cours de l'enquête, la personne interrogée indiquait à partir d’une liste numérotée des membres de son propre GAL, les personnes connues et non connues personnellement, les plus actives et les moins actives et celles avec qui la collaboration était souhaitée ou au contraire non désirée. L'échantillon des personnes enquêtées se composaient des membres du GAL (voir tableaux 1 et 2). formée dans le nouveau système et investie dans la vie locale par la création d'entreprises et/ou leur participation aux conseils municipaux.

Dans le GAL hongrois, l'intégration des membres au sein du réseau est plutôt faible car la plupart des personnes ne se connaissent pas. Trois personnes seulement sont connues et ont développé un degré de centralité élevé : le maire de Bóly (première micro-région) qui est le président du GAL, le député-maire de Mecseknádasd (seconde microrégion), et la manager du GAL qui est aussi responsable d'une association de danse folklorique. Le graphe montre une forte segmentation entre les individus en fonction de leur appartenance à l'une ou l'autre des micro-régions (Chevalier, 2012) (figure 4) ${ }^{8}$. Comme en Allemagne, on retrouve une rupture générationnelle entre ces acteurs. Il peut s'agir d'anciens cadres formés par le régime socialiste ou d'une nouvelle génération fortement investie dans la vie locale et qui tire généralement son prestige de son investissement en faveur du développement du territoire local. Généralement très bien formés aux problématiques du développement rural, ces acteurs constituent une élite locale, très réduite, composée d'élus et de managers qui semblent être les seuls à maîtriser le dispositif LEADER.

\section{Système d'action local et coordination de l'action de développement}

La projection cartographique du réseau de relations restitue la dimension territoriale de la polarisation du GAL autour des acteurs qui semblent jouer un rôle déterminant dans l'impulsion du développement local. La sociologie des réseaux les interprète comme des acteurs centraux.

Dans le cas du GAL situé dans les nouveaux Länder allemands, le pilotage de la démarche LEADER relève principalement du groupe des exploitants agricoles sociétaires d'un petit massif de moyenne montagne situé à l'ouest du périmètre d'intervention. Paradoxalement, l'initiative et l'implication viennent des marges montagnardes, de la périphérie de la région administrative - le district sur laquelle s'appuie aujourd'hui la région du pro-

\footnotetext{
8. Le traitement statistique et la représentation graphique des résultats de l'enquête par questionnaire ont été réalisés par Lala Razafimahéfa, ingénieur d'études au CNRS et par Jean-Claude Raynal, ingénieur d'études à l'EHESS.
} 
gramme LEADER. Ce petit massif déshérité, accolé à l'ancienne frontière interallemande et éloigné de tout, abrite une société locale dont les membres se sont montrés capables, dès le début des années 1990, de conduire l'adaptation des structures économiques et d'investir les dispositifs de développement local. Autour des entrepreneurs qui ont pris en charge la restructuration du secteur agricole s'est constitué un groupe d'acteurs qui réunit les responsables des associations chargées de missions d'aménagement ainsi que les promoteurs de la pépinière d'entreprises, dont le siège a été par la suite déplacé dans l'un des chefs-lieux du district (Schmalkalden). L'action publique est ensuite dynamisée par l'implication des maires des communes situées plus à l'est dans la vallée. Confrontés à la déprise des activités agricoles et industrielles, leur implication est liée aux prérogatives que la réforme territoriale leur a conférées à la suite de la restauration de l'autonomie locale et dans le cadre de structures de coopération intercommunale. C'est à cette échelle territoriale

Figure 3 : Les liens d'interconnaissance au sein du système d'action local - GAL Henneberger Land, Land de Thuringe, Allemagne Links of mutual acquaintance within the local action system - Henneberger Land Local Action Group, Land of Thuringia, Germany

Figure 4 : Les liens d'interconnaissance au sein du système d'action local GAL Zengő-Duna en Baranya (Hongrie) Links of mutual acquaintance within the local action system - ZengöDuna Local Action Group in Baranya, Hungary

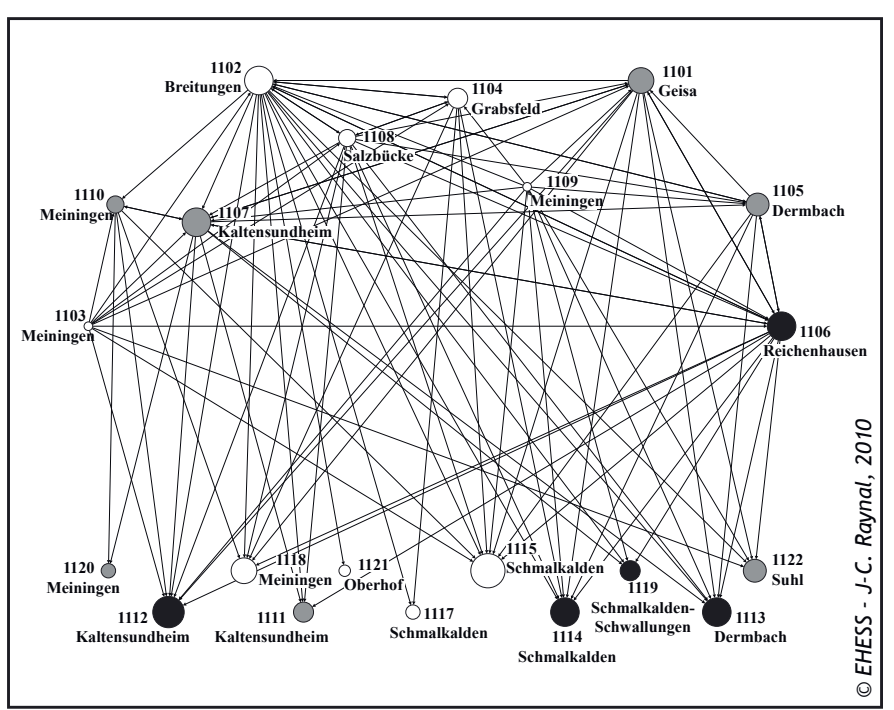

Indiquez la personne que vous connaissez le mieux
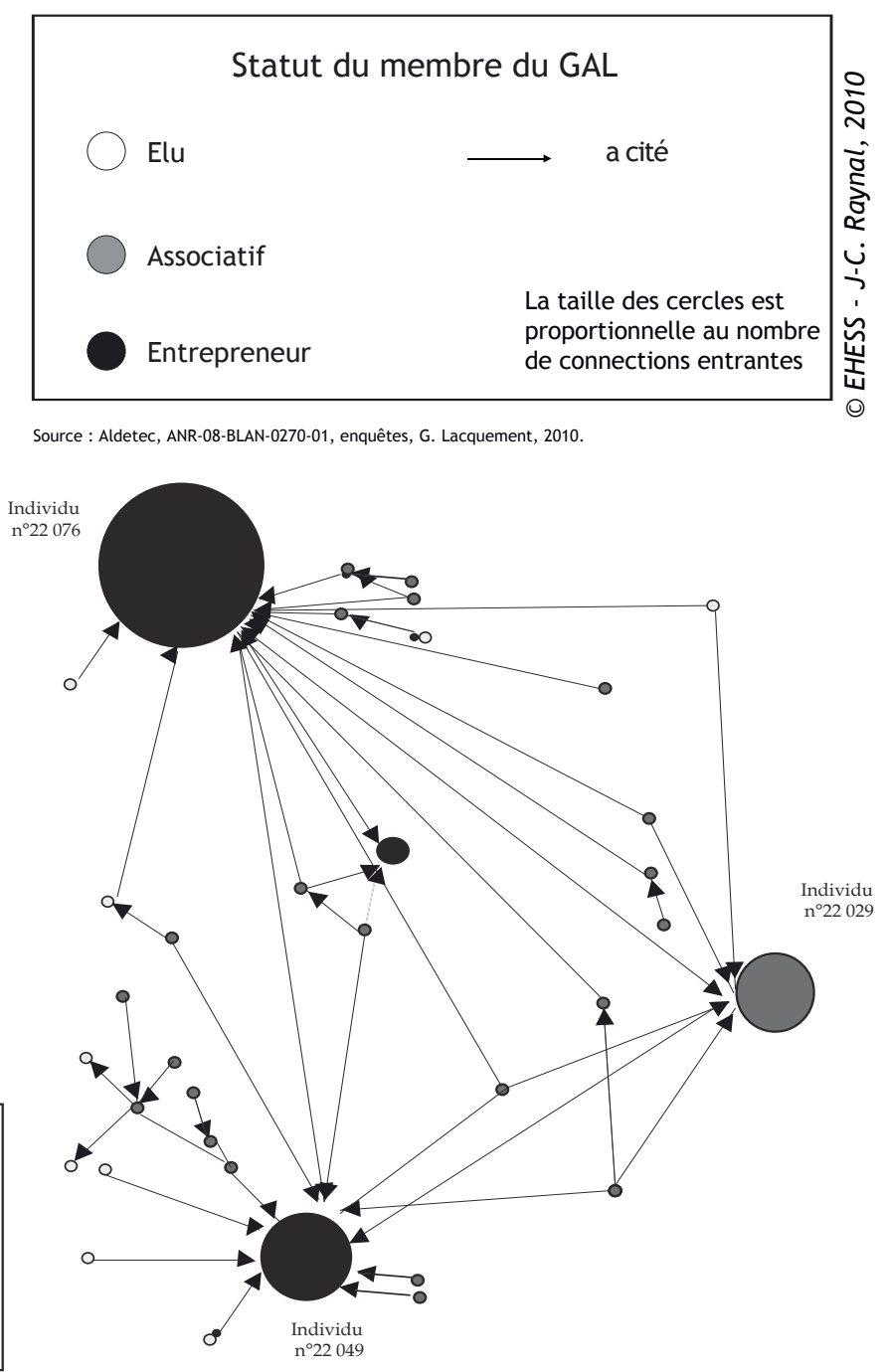

OP. Chevalier, MC. Maurel, P. Polà / ART-Dev UMR 5281 CNRS / EHESS / 2013 
que les projets sont conçus et mis en œuvre. La centralité du réseau de relations du GAL Henneberger Land s'organise donc en quelque sorte autour d'un groupe de pionniers qui ont conduit l'adaptation des activités économiques dans le contexte brutal de la transition et de la restructuration de l'économie socialiste (figure 5). Ces formes d'implication se traduisent par la distribution spatiale des projets de développement. La localisation des projets est révélatrice des formes de pilotage et de coordination de

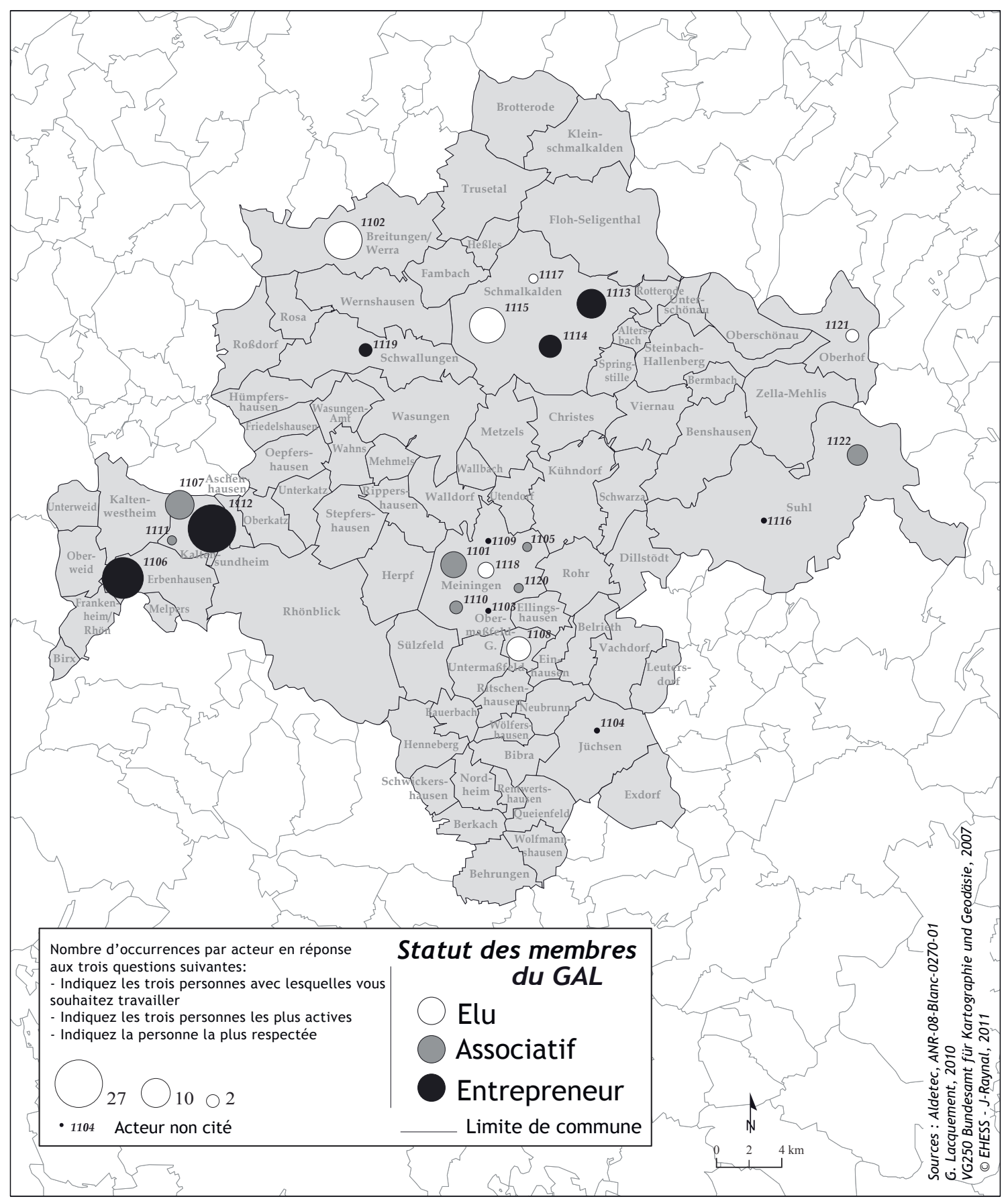

Figure 5 : Projection spatiale du degré de centralité des membres du GAL Henneberger Land, Land de Thuringe, Allemagne Germany Spatial projection of the centrality of members of the Henneberger Land Local Action Group, Land of Thuringia, 
l'action locale. Elle montre les lieux où les acteurs locaux se sont approprié le document stratégique du GAL et ont pu de cette manière concevoir des projets concrets de création de nouvelles activités économiques, de restauration du patrimoine rural, d'ouverture ou de maintien de services à la population (figure 6) (Lacquement et Raynal, 2013). Dans ce cas, la démarche participative apparaît polarisée,

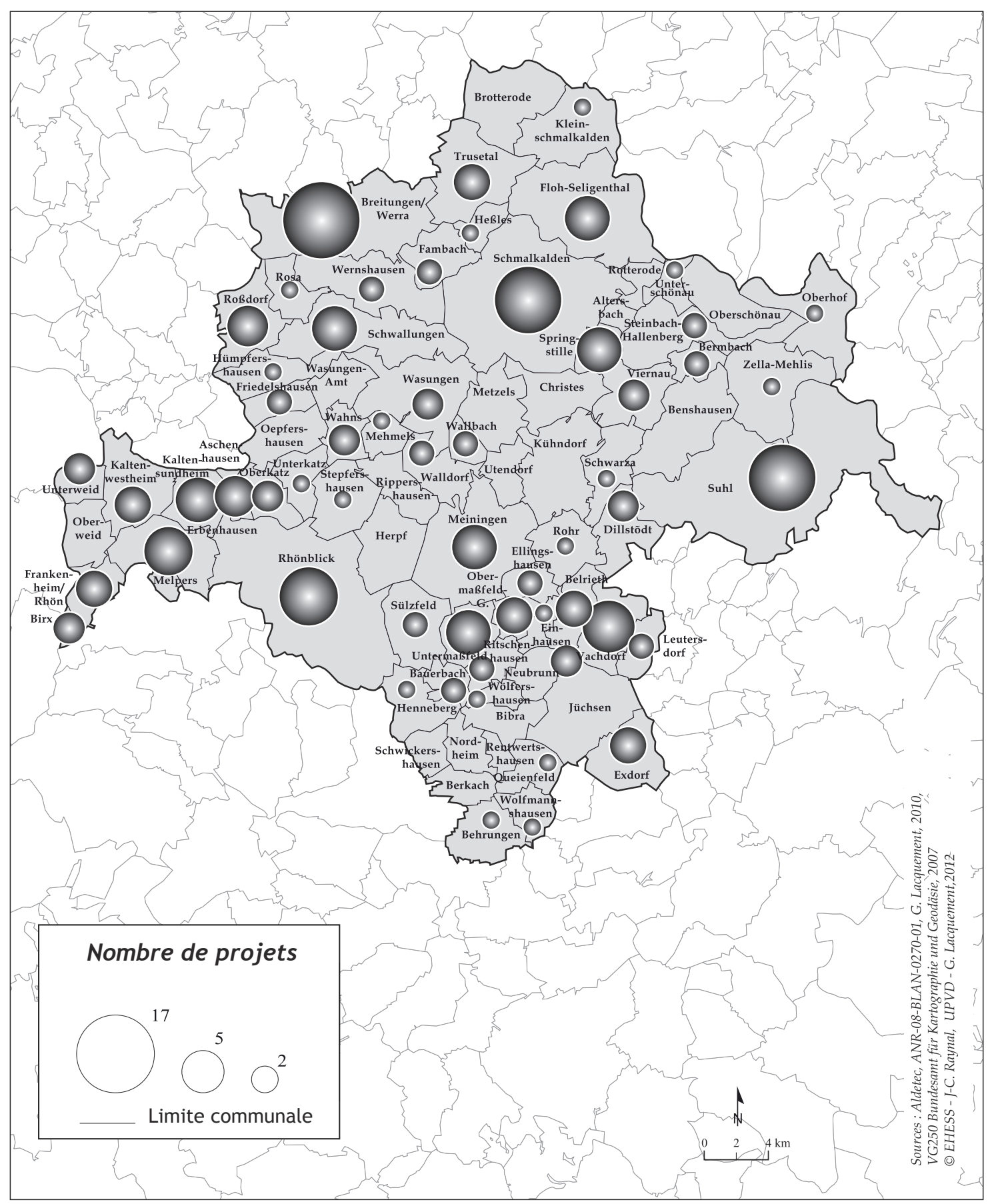

Figure 6 : Nombre et répartition des projets de développement conçus par le GAL Henneberger Land, Land de Thuringe, Allemagne

Number and distribution of development projects devised by the Henneberger Land Local Action Group, Land of Thuringia, Germany 
mais la densité des liens interpersonnels autour des acteurs centraux permet l'expression d'une force de proposition et de projet qui se distribue au bénéfice de l'ensemble du territoire local.

Dans le cas du GAL hongrois, la projection spatiale du degré de centralité confirme la configuration d'un réseau largement dominé par les membres de l'élite locale. Celle-ci comprend quelques maires et la manager du GAL. Le prestige et la notoriété se concentrent sur deux personnalités principales, chacune bien ancrée dans sa micro-région (figure 7). La répartition communale des projets se présente de manière dissymétrique (3/4 des projets sont localisés dans la micro-région de Mohács), mais plus encore la distribution des crédits alloués aux projets (figure 8). Cette dissymétrie reflète les déséquilibres démographiques et économiques entre les deux régions. Le contexte général est marqué par le dépeuplement et la déprise des activités rurales. Les difficultés de la reconversion économique maintiennent le chômage

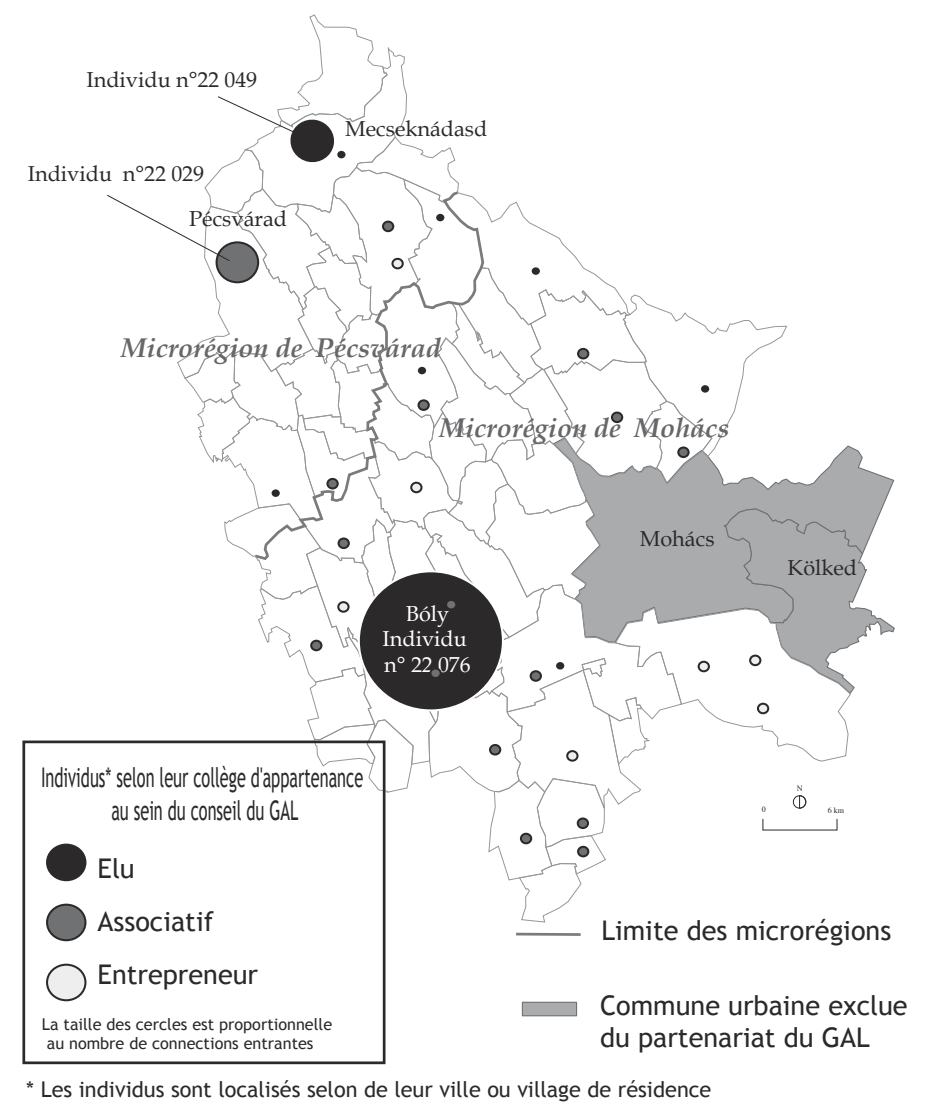

Figure 7 : Projection spatiale du degré de centralité des membres du GAL Zengö-Duna en Baranya, Hongrie

Spatial projection of the centrality degree of the members of the Zengö-Duna Local Action Group in Baranya, Hungary rural à un niveau élevé. Dans ce contexte, la micro région de Bóly se montre plus dynamique et a multiplié les projets de diversification dans les activités touristiques (labellisation et routes touristiques), les projets d'amélioration des services et de modernisation des équipements pour accroître l'attractivité résidentielle et économique. Mais cette dissymétrie révèle aussi l'existence d'un rapport de force politique qui se fait au bénéfice du maire de Bóly. La démarche participative est presque confisquée par les acteurs centraux et la fragmentation des liens interpersonnels au sein du réseau concentre l'action de développement au bénéfice de quelques communes.

Composé d'un grand nombre de membres, dépourvu de la cohésion fondée sur des liens d'interconnaissance, le partenariat local se structure donc autour d'une poignée d'élus locaux. Par leur rôle et leur position centrale au sein du réseau, les maires exercent un véritable leadership politique (Kovách, 2002), ce qui leur permet de faire jouer, de manière plus ou moins informelle, leurs réseaux politicoadministratifs pour capter l'information nécessaire au montage de leurs projets et pour obtenir des compléments de financement selon les opérations programmées. Ils sont les seuls en mesure de mobiliser l'énergie des autres acteurs, les compétences des professionnels de l'action publique (les managers qu'ils embauchent pour animer le travail), ainsi que les cofinancements indispensables à la réalisation des projets. Ils sont convaincus de servir les intérêts de leur collectivité locale. Ce sont eux qui, prenant pour intermédiaires les associations placées sous leur protection, entreprennent de définir et dans certains cas de réinventer l'identité culturelle locale à partir d'une approche muséographique des vestiges hérités (églises, temples, cimetières, monuments publics).

Le mode d'exercice paternaliste du leadership politique exerce un effet inhibiteur sur l'apprentissage de l'engagement civique. Pour beaucoup de membres de l'assemblée du GAL, l'implication dans le fonctionnement du partenariat est limitée. Même si la société locale est tenue informée par le biais des forums de discussion et des réunions publiques, force est de constater qu'elle s'investit peu dans l'action collective et les projets d'aménagement. Dans ces conditions, le réseau d'acteurs tel qu'il a été constitué ne favorise pas une authentique participation des habitants. Prenant appui sur les 
acteurs les plus dynamiques, les projets ne prêtent pas une grande attention aux groupes marginalisés. C'est ainsi que les Roms, nombreux dans les villages du Baranya, se trouvent de fait exclus alors que les représentants de la minorité souabe, parmi lesquels se recrutent les entrepreneurs mais aussi les responsables des associations culturelles les plus actives, font figure d'élite sociale privilégiée. Seuls les acteurs qui peuvent mobiliser leur savoir-faire et leurs relations pour accéder à l'information sont en situation d'inclusion au sein du GAL. Ceci tend à renforcer la «classe de projet », formée par ceux qui gravitent autour de ces démarches de développement local (Kovách et Kučerova, 2006). Présentée comme un vecteur d'amélioration de la gouvernance locale, l'action LEADER ne favorise pas l'émergence d'un partenariat intégrateur.

L'exercice de comparaison montre au final qu'un lien de causalité s'établit entre l'implication relative des individus dans la démarche collective et la configuration des structures spatiales issues de deux décennies de transformation post-socialiste. Le fonctionnement des GAL n'est pas étranger aux effets de lieu. La transposition du modèle LEADER semble dépendante du contexte géographique de réception. Les formes différenciées d'ancrage des GAL dans les territoires locaux montrent que les facteurs spatiaux interviennent dans les jeux sociaux et que les stratégies développées par les acteurs sociaux dépendent de propriétés spécifiques aux lieux et à l'organisation de l'espace (Rey, 1996; Von Hirschhausen, 2006). Ces propriétés ne déterminent pas a priori les stratégies d'acteurs mais constituent un potentiel territorial qui peut être plus ou moins valorisé par les acteurs sociaux (Maurel, 2005). L'implication relative des élus s'explique par les difficultés qui continuent de peser sur l'adaptation des activités économiques. Le démantèlement des structures de l'économie socialiste au début des années 1990, a provoqué une déprise générale de l'activité et l'apparition du chômage rural a entraîné l'émigration des forces vives. Le délitement du tissu économique n'a néanmoins pas les mêmes effets dans les localités villageoises où domine l'activité agricole et dans les villes moyennes et les bourgscentres qui, malgré l'ampleur de la crise et les difficultés de la reconversion, tendent à polariser les nouvelles activités de service. Dans ce contexte,

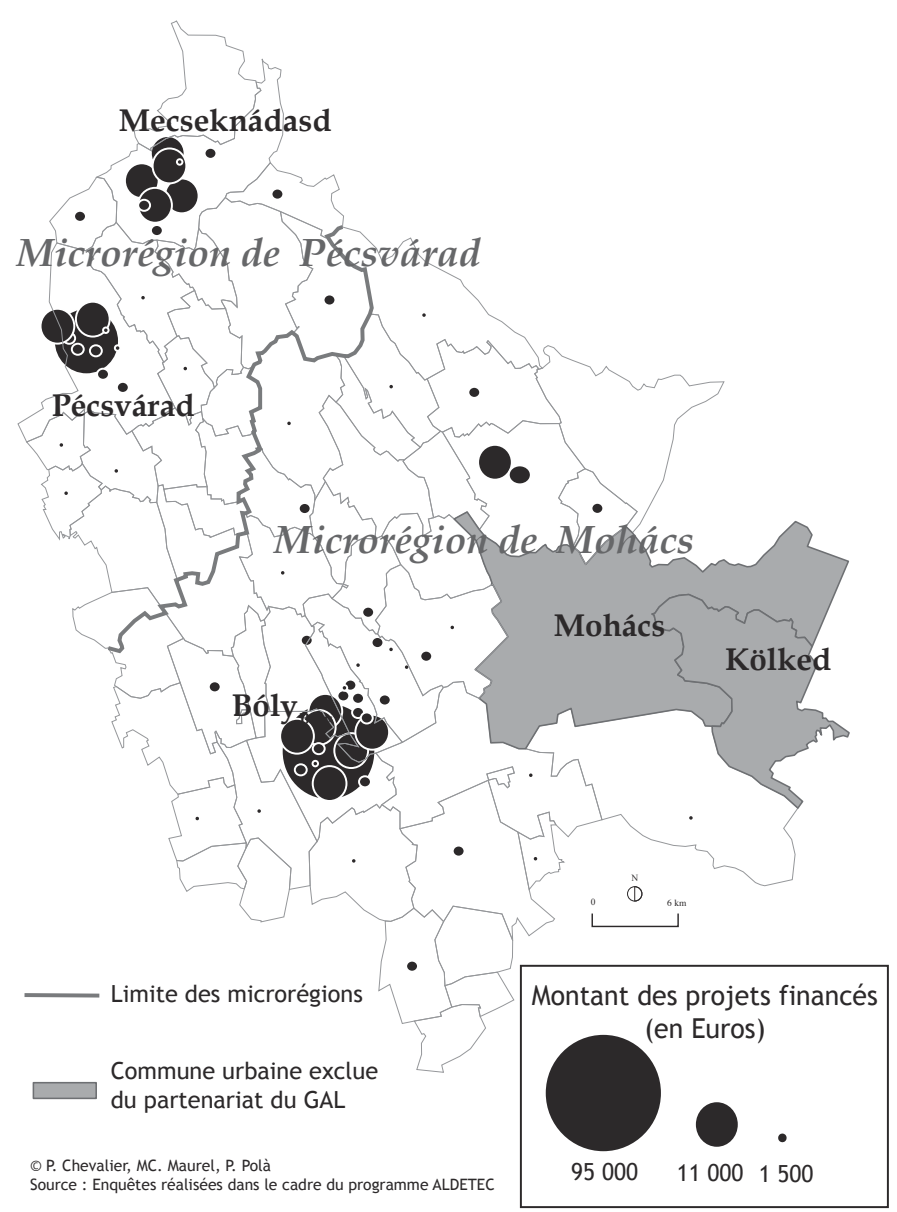

Figure 8 : Montants accordés aux projets de développement conçus par le GAL Zengö-Duna en Baranya, Hongrie

Sums granted to development projects devised by the Zengö-Duna Local Action Group in Baranya, Hungary

les formes de coordination de l'action collective révèlent la concentration du pouvoir d'intervention entre les mains des élus dont les stratégies d'action se répercutent sur l'organisation du territoire local à travers la mise en œuvre des projets LEADER (Lacquement et Raynal, 2013; Chevalier, Maurel et Pola, 2015). Le maintien de la grande exploitation comme structure de production dominante dans le secteur agricole renforce le poids des chefs d'exploitation sociétaire dans les GAL, comme partenaires quasi exclusifs de la sphère économique. Enfin, les modalités d'application des réformes territoriales et de transfert de prérogatives aux échelons locaux jouent pleinement sur les formes de coordination de l'action locale, souvent au prix d'un renforcement des processus élitaires. 


\section{Conclusion}

En Europe centrale, le programme LEADER constitue une nouveauté institutionnelle dans le sens où il participe à l'introduction d'un mode nouveau de gouvernance des territoires locaux. Cette nouveauté institutionnelle est référée à un modèle construit sur les paradigmes du développement local et de la gouvernance locale. La diffusion de l'innovation se lit dans l'aptitude des acteurs locaux à organiser des partenariats ou des réseaux de coopération et à délimiter des périmètres d'intervention ou d'action. Ce sont ces partenariats qui prennent en charge la coordination de l'action locale de développement par la conception de stratégies ainsi que par la réalisation de projets concrets.

Comme le montre l'approche comparative de l'application du programme LEADER, la diffusion de cette innovation est différenciée par deux facteurs principaux. Le premier renvoie aux prismes nationaux qui encadrent la démarche LEADER par des logiques qui oscillent entre la logique d'accompagnement et la logique d'intervention. Ces logiques se rattachent à des trajectoires institutionnelles de gestion du territoire qui intègrent de manière plus ou moins prononcée les processus de décentralisation et de redistribution des prérogatives d'aménagement à l'échelon local. Le second tient à la structuration et au fonctionnement des réseaux de coopération qui portent l'action de développement. Ces réseaux se montrent sensibles aux effets de lieux et de contextes qui tendent à singulariser la trajectoire d'évolution des territoires post-socialistes.

\section{Bibliographie}

Chevalier P., Maurel M.-C., Pola P., 2015. Le programme LEADER en Hongrie, un pilotage de l'action publique sous contrôle, Revue du Bulletin de la Société de Géographie de Liège (BSGL), nº 64/2015, p. 37-51.

Chevalier P. (dir.), 2012. Le modèle européen LEADER (2007-2013), Logiques d'action et contextes de réception, Revue d'Etudes Comparatives Est-Ouest, no 3, 197 p.

Chevalier P., Maurel M.-C., 2013. The LEADER programme in central Europe: a new local development policies (Program LEADER w Europies Centralnej. Nowa polityka rozwoju lokalnejo), in Psyk-Piotrowskiej E., Zajda K. (dir.), Podejści LEADE - inne formy aktywizacji - Społecznośch Folia Sociologica, n ${ }^{\circ} 44$, p. 39-52
Commission Européenne, 2011 . Rapport de la commission au Parlement européen, au Conseil, au Comité économique et social européen et au Comité des régions concernant la mise en œuvre des plans stratégiques nationaux et orientations stratégiques de la Communauté pour le Développement Rural (période de programmation 2007-2013), Publication de la communauté européenne, no 943, Bruxelles, 122 p.

Cloutier J., 2003. Qu'est-ce que l'innovation sociale?, Cahiers $d u$ CRISES, Collection Études théoriques, $\mathrm{n}^{\circ}$ ET0314, $60 \mathrm{p}$.

Dargan L., Shucksmith M., 2008. LEADER and Innovation, Sociologia Ruralis, vol. 38, n 3, p. 274-291.

Delpeuch T., 2008. L'analyse des transferts internationaux de politiques publiques : un état de l'art, Questions de recherche, CERI/Sciences Po, Paris, n² 27, 69 p.

Dubois J., 2009. Les politiques publiques territoriales, la gouvernance multi-niveaux face aux défis de l'aménagement, Rennes, PUR, 216 p.

Duran P., 2001. Action publique, action politique, in LERESCHE J.-P. (dir.), Gouvernance locale, coopération et légitimité, Paris, Pedone, p. 369-389.

Dolowitz D., Marsh D., 2000. Learning from Abroad: The Role of Policy Transfer in Contemporany Policy Making, Governance, vol. 13, nº 1, p. 5-24.

FonTAN J.-M. et al., 2004. Innovation et société, pour élargir l'analyse des effets territoriaux de l'innovation, Géographie, économie, société, vol. 6, n 2, p. 115-128.

Forsé M., 2008. Définir et analyser les réseaux sociaux, les enjeux de l'analyse structurale, Informations sociales, vol. 3, $n^{\circ} 147$, p. 10-19.

Hillier J. et al., 2004. Trois essais sur le rôle de l'innovation sociale dans le développement territorial, Géographie, économie, société, vol. 6, n 2, p. 129-152.

Hirschhausen B. von, 2006.Trajectoires de décollectivisation en Roumanie, questionner les héritages à partir des différences géographiques, in Kотт S., Mespoulet M. (dir.), Le post-communisme dans l'histoire, PUB, Bruxelles, p. 127-143.

KovÁch I., 2002. Leadership, Local Power and Rural Restructuring in Hungary, in Halfacree K., Kovách I., Woodmard R. (dir.), Leadership and Local Power in European Rural Development, Aldershot, Ashgate, Burlington, Sidney, Singapore, p. 91-144.

Kovách I, Kučerova E., 2006. The Project Class in Central Europe: The Czech and Hungarian Cases, Sociologia Ruralis, vol. 46, nº 1, p. 3-19.

Lacquement G., 2012. Pratiques de la gouvernance locale en Allemagne orientale : transfert institutionnel, apprentissage social et logiques réticulaires dans le cadre du programme européen LEADER 2007-2013, in Chevalier P. (dir.), Le modèle européen LEADER (2007-2013) : Logiques d'action et contextes de réception, Revue d'études comparatives EstOuest, vol. 43, n 3, p. 57-89.

Lacquement G., Raynal J.-C., 2013. Acteurs et ressources du développement local en Allemagne orientale : le territoire rural au prisme des projets du programme européen LEADER, Annales de Géographie, no 692, p. 393-421. 
Lebart L., Morineau A., Piron M., 1997. Statistique exploratoire multidimensionnelle, Dunod, Paris, 439 p.

Le Gales P., 2010. Gouvernance, in Boussaguet L., Jacquot S., Ravinet P. (dir.), Dictionnaire des politiques publiques, Paris, Les Presses de Sciences Po, p. 244-251.

Leloup F., Moyart L., Pecqueur B., 2005. La gouvernance territoriale comme nouveau mode de coordination territoriale?, Géographie, Economie et Société, vol. 7, n 4, p. 321-331.

LiN N., 1995. Les ressources sociales : une théorie du capital social, Revue Française de Sociologie, vol. 36, n 4, 1995 , p. 685-704.

Maurel M.-C., 2005. Temps de recomposition des territoires ruraux en Europe centrale, Revue d'Études Comparatives Est-Ouest, vol. 36, n 2, p. 5-38.

Maurel M.-C., 2008. L'action publique «par le bas » : l'approche LEADER en Europe centrale, Revue d'études comparatives Est-Ouest, vol. 39, n 4, p. 33-61.
Maurel M.-C., Chevalier P., Lacquement G., 2014. Transfert et apprentissage du modèle LEADER en Europe centrale, Paris, L'Harmattan, coll. «Questionner l'Europe », 300 p.

Mercklé P., 2011 . Sociologie des réseaux sociaux, Paris, La Découverte, nouvelle édition, 128 p.

REY V. (dir.), 1996. Les nouvelles campagnes d'Europe centrale et orientale, Paris, CNRS, 240 p.

Teisserenc P., 1994. Politique de développement local, la mobilisation des acteurs, Sociétés Contemporaines, no 18-19, p. 187-213.

TiETz A. (dir.), 2007. Ländliche Entwicklungsprogramme 20072013 in Deutschland im Vergleich, Finanzen, Schwerpunkte, Massnahmen, Landbauforschung Völkenrode - Agricultural Research, Bundesforschungsanstalt für Landwirtschaft (FAL), Braunschweig, 174 p. 